\title{
WiseMAC: An Ultra Low Power MAC Protocol for the Downlink of Infrastructure Wireless Sensor Networks
}

\author{
A. El-Hoiydi and J.-D. Decotignie \\ CSEM, Swiss Center for Electronics and Microtechnology, Inc. \\ Rue Jaquet-Droz 1, 2007 Neuchâtel, Switzerland \\ Emails: $\{$ aeh,jdd $\} @$ csem.ch
}

\begin{abstract}
This paper proposes WiseMAC (Wireless Sensor MAC) for the downlink of infrastructure wireless sensor networks. WiseMAC is a novel energy efficient medium access control protocol based on synchronized preamble sampling. The trade-off between power consumption and delay is analyzed, focusing on low traffic. WiseMAC is compared analytically with the power management protocol used in the IEEE 802.15.4 ZigBee standard. It is shown that WiseMAC can provide a significantly lower power consumption for the same delay. ${ }^{1}$
\end{abstract}

\section{Introduction}

Inexpensive integrated system-on-a-chip devices comprising a radio transceiver and a microcontroller have been since a few years a subject of research [1]. Industry is now selling such devices [2]. They will permit to implement ubiquitous computing applications where small batterypowered nodes are interconnected via a wireless network. As now widely recognized, one of the main issues is the power consumption of such devices. A meticulous design of the medium access control (MAC) protocol is key to reach a low power consumption.

\subsection{Problem statement}

Unlike most research dealing with wireless sensor networks [3], we do not consider an ad-hoc network topology, but an infrastructure network. An infrastructure network is composed of a number of access points interconnected through a backbone network. Each access point is serving a number of sensor nodes. Such a topology can be envisaged for example in smart building applications, where the Ethernet or powerline cabling can be used for the backbone network. The main characteristic of access points is that they

\footnotetext{
${ }^{1}$ The work presented in this paper was supported in part by the National Competence Center in Research on Mobile Information and Communication Systems (NCCR-MICS), a center supported by the Swiss National Science Foundation under grant number 5005-67322.
}

are usually energy unconstrained. This fact is exploited by the MAC protocols under consideration in two ways: first, an access point can listen continuously to potential uplink traffic, and secondly, an access point may send any amount of signalling traffic for free (e.g. beacons, wake-up signal). This work also applies to other systems where energy unconstrained nodes can play the role of base stations, such as clustered ad-hoc networks with solar powered cluster heads [4] or vehicle mounted mobile access points moving through a cloud of sensors to collect data.

An energy efficient wireless MAC protocol is a protocol that minimizes idle listening and overhearing [5]. In addition, as any other MAC protocol, it should keep to a minimum collisions and protocol overhead. Idle listening refers to the active listening to an idle channel, waiting for a potential packet to arrive. Overhearing refers to the reception of a packet, or of part of a packet, that is destined to another node. As the power consumption of a transceiver in receive mode is far from being negligible, idle listening may become the main source of energy waste, especially in low traffic conditions. To reach a low average power consumption, the transceiver must be shut down most of the time (i.e. duty cycling).

In infrastructure networks, one must distinguish the downlink (from the access point to the sensor nodes) from the uplink (from the sensor nodes to the access point). For each direction, a different radio channel and/or a different MAC protocol may be used.

In the downlink direction, the challenge is to transmit data from the access point to some sensor node, without requiring that the sensor node continuously listens to the channel. A trade-off must be made between power consumption and transmission delay.

The problem is different in the uplink direction. As the access point is not energy limited, it can listen all the time to the channel. The issue to resolve in the uplink is the multiple access to a shared medium. If the system is operated near channel capacity, this problem is very complex. However, if only a moderate traffic is present on the channel, finding a energy efficient uplink MAC protocol is relatively 
easy. For example, the simple non-persistent CSMA protocol [6] can approach the ideal case for power conservation, with no idle listening, no overhearing and little collisions.

In an infrastructure wireless sensor network under low traffic load, the main issue is hence the design of the downlink MAC protocol. In this paper, we therefore focus on the downlink problem.

Protocols for uplink and downlink may be designed jointly. Uplink traffic can be exploited to enhance the performance of the downlink protocol. For example, an uplink packet can carry a request to transmit potentially buffered data in the downlink direction. Nevertheless, a stand-alone downlink MAC protocol is needed to guarantee a given transmission delay in periods during which uplink traffic is absent.

Sensor networks are usually meant for the acquisition of data. Most traffic can be expected in the uplink channel. The downlink channel is foreseen to carry configuration and querying requests. With such a traffic, inter-arrivals measured in minutes or hours will be common. We will assume throughout this paper that the inter-arrival between packets is much larger than the time needed to transmit a packet.

\subsection{Related work}

A large effort has been devoted by the research community to the development of medium access control protocols for wireless computer networks $[6,7]$. Such protocols have been primarily designed to minimize the transmission delay and to maximize the throughput. The power consumption has only later become an issue of large interest. In [8], a comparison between the power consumption of several wireless MAC protocols is given. In their analysis, Chen et al. focused on high traffic situations. In this paper, we focus, on the contrary, on low traffic situations. Light traffic is expected to be very common in certain applications of ubiquitous computing, where a very long lifetime is required. It is therefore necessary to understand and minimize the energy consumption of MAC protocols under light traffic conditions.

Research on ultra low power MAC protocols for ad-hoc wireless sensor network has started a few years ago. A number of proposals are available, among which one can cite S-MAC [5] and STEM [9]. Protocols for ad-hoc sensor networks can potentially be interesting for the downlink of infrastructure sensor networks. The work presented in this paper differs from previous research on ad-hoc sensor networks, mainly because we focus on an infrastructure topology, and investigate how the unconstrained energy supply of the access point can be exploited.

Paging systems bear similarities with infrastructure sensor networks. Several standards have been developed over the years, e.g. POCSAG and FLEX [10]. The techniques used by paging systems are useful sources of inspiration, but these protocols cannot be used as such for infrastructure wireless sensor networks. Paging protocols seek the capacity-energy optimum, while we seek the delay-energy optimum.

IEEE 802.15.4 is a standard for Low Rate Wireless Personal Area Networks (LR-WPANs) [11]. It is often referred to as ZigBee, the name of the industrial alliance promoting it. The ZigBee protocol is very well suited to address, in an energy efficient manner, the problem of downlink traffic in infrastructure networks. WiseMAC will be compared to the power save MAC protocol used in ZigBee.

\subsection{Original contributions}

The contribution of this paper is the proposal of WiseMAC (Wireless Sensor MAC), a novel protocol for the downlink of infrastructure networks. This protocol has been briefly outlined in [12] for multi-hop sensor networks. We show here that it is also of interest for infrastructure sensor networks. WiseMAC is based on the preamble sampling technique to minimize idle listening [13]. The novel idea in WiseMAC is to minimize the length of the wake-up preamble, exploiting the knowledge of the sensor nodes sampling schedules. Initial results of the study of WiseMAC in infrastructure networks were presented in [14]. This paper presents and analyzes an improved version of WiseMAC, where data frames are repeated in long preambles to mitigate overhearing and thereby achieve scalability.

The rest of the paper is organized as follows: Section 2 describes WiseMAC and Section 3 the power save protocol of ZigBee. A performance comparison is done in Section 4. Section 5 gives concluding remarks.

\section{WiseMAC}

WiseMAC is based on the preamble sampling technique [13]. This technique consists in regularly sampling the medium to check for activity. By sampling the medium, we mean listening to the radio channel for a short duration, e.g. the duration of a modulation symbol. All sensor nodes in a network sample the medium with the same constant period $T_{W}$. Their relative sampling schedule offsets are independent and constant ${ }^{2}$. If the medium is found busy, a sensor node continues to listen until a data frame is received or until the medium becomes idle again. At the access point, a wake-up preamble of size equal to the sampling period is transmitted in front of every data frame to ensure that the receiver will be awake when the data portion of the packet arrives. This technique provides a very low power consumption when the channel is idle. The disadvantages of this technique are that the (long) wake-up preambles cause a

\footnotetext{
${ }^{2}$ To be more precise, the relative sampling schedule offsets are constant up to the slow drift caused by quartz inaccuracy on each node.
} 


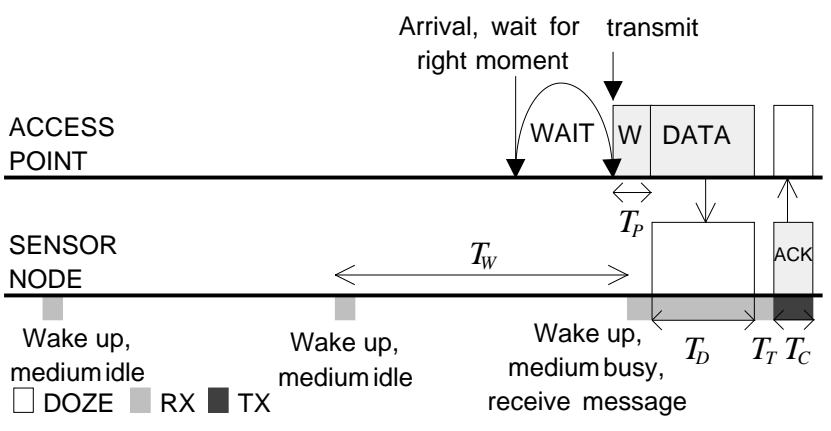

Figure 1. WiseMAC.

throughput limitation and a large power consumption overhead in reception. The overhead in reception is not only born by the intended destination, but also by all other nodes overhearing the transmission.

The novel idea introduced by WiseMAC consists in letting the access point learn the sampling schedule of all sensor nodes. Knowing the sampling schedule of the destination, the access point starts the transmission just at the right time with a wake-up preamble of minimized duration $T_{P}$ as illustrated in Fig. 1. The access point keeps an up-todate table with the sampling schedule of all sensor nodes. The sampling schedule information is gained through the inclusion in every acknowledgement packet of the remaining time until the next scheduled sampling.

The duration of the wake-up preamble must be computed such as to compensate for the drift between the clock at the access point and on the sensor nodes. This drift is proportional to the time since the last re-synchronization (i.e. the last time an acknowledgement was received from a given sensor node). Let $\theta$ be the frequency tolerance of the timebase quartz and $l$ the interval between two communications. The required duration of the wake-up preamble is

$$
T_{P}=\min \left(4 \theta l, T_{W}\right)
$$

This expression is obtained as follows: Assume that the access point has received fresh timing information from some sensor node at time 0 , and that the access point wants to send a packet to this sensor node at the node sampling time $l$. If the sensor node quartz has a real frequency of $f(1+\theta)$ instead of $f$, its clock will have an advance of $\theta l$ at time $l$. It is hence needed to start the preamble $\theta l$ in advance. Because the clock of the access point might be late, it must target a time $2 \theta l$ in advance. Because the clock of the access point might be early, and the clock of the sensor node late, the duration of the wake-up preamble must be of $4 \theta l$. If $l$ is very large, $4 \theta l$ may be larger that the sampling period $T_{W}$. In those cases, a preamble of length $T_{W}$ is used. We thus obtain (1).

If the traffic is high, the interval $l$ between communications will be small, and so the wake-up preamble ( $4 \theta l)$. If

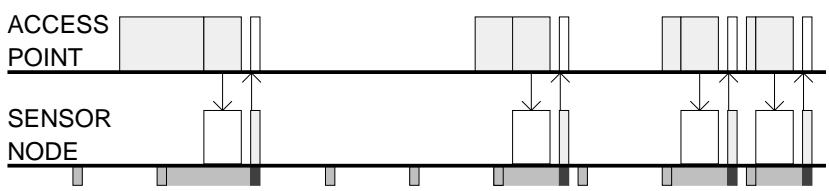

\section{Figure 2. Adaptivity of the per-packet over- head to the traffic.}

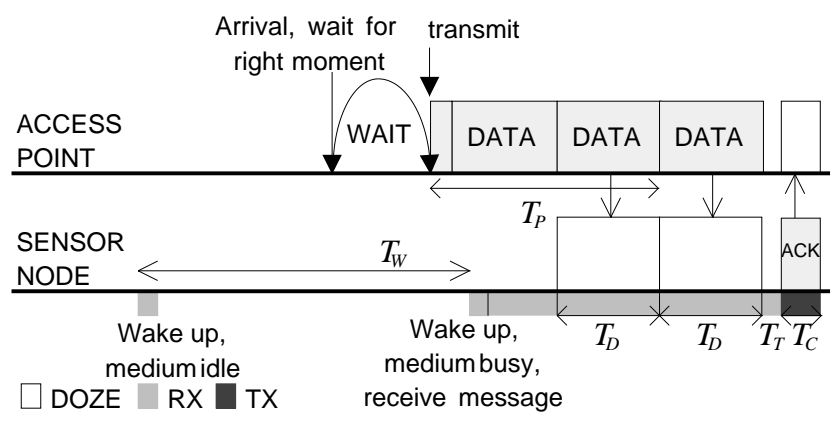

\section{Figure 3. WiseMAC - Repetitions of the data frame in long wake-up preambles.}

the traffic is low, the interval between communications will be large in average, but at maximum equal to $T_{W}$. This important property, illustrated in Fig. 2, makes the WiseMAC protocol adaptive to the traffic. The per-packet overhead decreases in high traffic condition.

Overhearing is naturally mitigated when the traffic is high, thanks to the combined use of the preamble sampling technique and the minimization of the wake-up preamble duration. As already mentioned, sensor nodes are not synchronized among themselves. Their relative sampling schedule offsets are independent. In high traffic conditions, the duration of the wake-up preamble being smaller than the sampling period, short transmission are likely to fall in between sampling instants of potential overhearers ${ }^{3}$.

When the traffic is low, the length of the wake-up preamble can exceed the length of the data packet. In such cases, the wake-up preamble is composed of padding bits followed by repetitions of the data frame, as shown in Fig. 3. A sensor node that finds the medium busy will wait for the start of frame delimiter located at the beginning of each data frame. In the header of the data frame, this sensor node will see whether the packet is destined to itself or not. If the destination is another node, it goes back to sleep. If the destination is itself, it waits for the end of the transmission and sends an acknowledgement.

An important detail of the WiseMAC protocol, which

\footnotetext{
${ }^{3}$ To be effective, the probabilistic overhearing mitigation requires the sampling period to be much larger than the packet duration. This condition is met in most practical situations.
} 
was inspired by the IEEE 802.11 [15] and IEEE 802.15.4 ZigBee protocols, is the presence of a frame pending bit in the header of data packets. A sensor node receiving a data packet with this bit set will continue to listen after having sent the acknowledgement. The next packet will be sent by the access point right after having received the acknowledgement. This scheme permits to use a wake-up interval that is larger than the average interval between the arrivals for a given node. It permits to reduce the queuing delay at the access point, especially in the event of traffic bursts.

Finally, it is interesting to note that collisions are not possible using WiseMAC for a downlink channel, as the access point is the only initiator of communications.

\section{IEEE 802.15.4 ZigBee}

An IEEE 802.15.4 ZigBee network includes a central coordinator. We will call this node the access point, assuming it is connected to the fixed network and supplied with an unconstraint amount of energy. A power save scheme has been specified in the IEEE 802.15.4 standard to save energy at the cost of a larger delay (the same scheme was already selected for IEEE 802.11). The access point buffers incoming traffic addressed to sensor nodes. A beacon is periodically transmitted with period $T_{W}$. This beacon contains the address of sensor nodes for which data packets have been buffered. All sensor nodes wake-up regularly to receive the beacon. If they discover their address in it, they poll the access point to receive the buffered data.

The standard requires the access point to reply to a poll packet after a given delay. In practice, it is difficult for the access point software to find the right data packet and prepare it for transmission within the specified delay. Instead, the access point replies to the poll packet with an ack packet. This instructs the sensor node to remain in listening mode. As soon as possible, the access point sends the data packet, which in then acknowledged back by the sensor node. In summary, the polling procedure is composed of four packet transmissions: POLL-ACK-DATA-ACK. In this paper, we are interested in the basic performance of a protocol that would use such a traffic indication beacon. For a fair comparison with WiseMAC, we consider a version of the ZigBee protocol, that is fully optimized for low power operation. We assume first that access point replies to a poll packet with a data packet and secondly that a data packet is not acknowledged immediately. The acknowledgement is piggy-backed on the following poll packet. This procedure is illustrated in Fig. 4.

With ZigBee, the header of data packets contain a 'frame pending' bit indicating the presence of more data packets waiting at the access point. This indicates to the sensor node that it must poll the access point again to download the following data packet.

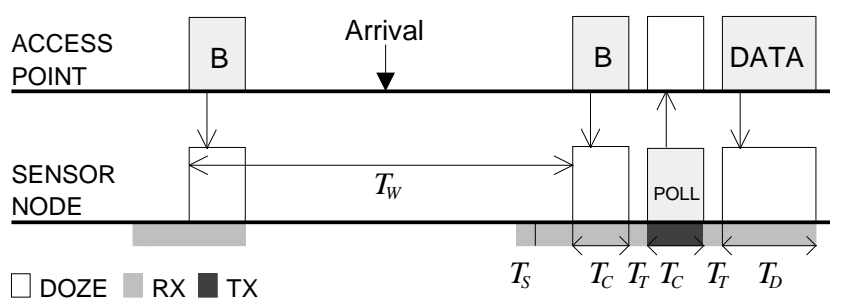

Figure 4. IEEE 802.15.4 ZigBee Power Save Protocol.

\section{Performance Analysis}

\subsection{Radio Model}

When considering low power protocols, it is very important to model precisely the transition delays between the different states of a transceiver and the power consumption in those states. The following states can be identified:

DOZE The transceiver is not able to transmit nor receive, but is ready to quickly power-on into the receive or the transmit state,

$\mathbf{R X}$ The transceiver is listening to the channel, receiving data or trying to demodulate data out of a noisy or idle channel,

TX The transceiver is transmitting data.

We denote with $T_{S}$ the setup time required to turn on the transceiver into the RX or the TX states, starting from the DOZE state. We denote with $T_{T}$ the turn-around time which is required to switch the transceiver between the RX and TX states. We denote with $B$ the bit rate of the transceiver.

The power consumed in the states DOZE, RX and TX states will be denoted, respectively, with $P_{Z}, P_{R}$ and $P_{T}$. During the phases when the transceiver is setup (or turnedaround) into the RX or TX states, we assume that the transceiver consumes a power of $P_{R}$.

To simplify analytical expressions, we define $\widehat{P}_{R}=$ $P_{R}-P_{Z}$ as the increment in power consumption caused by being in the RX state (as compared to the DOZE state), and $\widehat{P}_{T}=P_{T}-P_{Z}$ as the increment in power consumption caused by being in the TX state.

\subsection{Traffic Model}

We consider a population of $N$ sensor nodes under the responsibility of one access point. Downlink Poisson traffic arrives at the access point from the fixed network at a global rate $\lambda$. We assume that an equal part $\frac{\lambda}{N}$ of this traffic is destined to each sensor node. A given sensor node will receive data packets with an average packet inter-arrival time of $L=\frac{N}{\lambda}$. 
Data packets have a constant duration $T_{D}$. Control packets (pollings, acknowledgements, traffic indication map beacons) have a constant duration $T_{C}$.

As mentioned in the introduction, we assume a low traffic, where the global inter-arrival $1 / \lambda$ is much larger than the sum of the lengths of a data packet, of the turn-around time and of a control packet:

$$
1 / \lambda \gg T_{D}+T_{T}+T_{C}
$$

\subsection{Power Consumption and Delay}

This section introduces analytical expressions to compute the power consumption and the latency with WiseMAC and ZigBee under the low traffic assumption. Due to space limitations, no detailed explanations can be given on the derivation of those expressions. Interested readers are referred to [16] for details. It can be shown that the average power consumed by the WiseMAC and ZigBee protocols is respectively given by

$$
\begin{aligned}
P_{\text {WiseMAC }}= & P_{Z}+\frac{\widehat{P}_{R}\left(T_{S}+1 / B\right)}{T_{W}} \\
& +\frac{\widehat{P}_{R}\left(\bar{X}+T_{D}+T_{T}\right)+\widehat{P}_{T} T_{C}}{L} \\
& +\widehat{P}_{R}(N-1) \frac{\bar{Y}}{L}
\end{aligned}
$$

where

$$
\begin{aligned}
& \bar{X}=2 \theta L\left(1-e^{-\frac{T_{D}}{4 \theta L}}\right) \\
& \bar{Y}=\frac{T_{D}^{2}+12 T_{D} \theta L}{2 T_{W}}\left(1-e^{-\frac{T_{W}}{4 \theta L}}\right)
\end{aligned}
$$

and

$$
\begin{aligned}
P_{Z i g B e e}= & P_{Z}+2 \theta \widehat{P}_{R}+\frac{\widehat{P}_{R}\left(T_{S}+T_{C}\right)}{T_{W}} \\
& +\frac{\widehat{P}_{T} T_{C}+\widehat{P}_{R}\left(T_{D}+2 T_{T}\right)}{L}
\end{aligned}
$$

Expression (3) is composed of the power consumed in the DOZE state, of the power consumption increments caused by the preamble sampling activity (setup and listen during the duration of a radio symbol), the reception of the packet and the overhearing of this packet by $N-1$ neighbors. $\bar{X}$ is the duration during which a destination sensor node listens to the wake-up preamble prior to detect the start of the first data frame copy ${ }^{4} . \bar{Y}$ is the average duration

\footnotetext{
${ }^{4}$ With a periodic traffic (of period $L$ ), the average duration during which a destination listens to the wake-up preamble would simply be $\min \left(2 \theta L, T_{D} / 2\right)$, a result that is more intuitive. Expression (4) is obtained by taking the expectation of $\min \left(2 \theta l, T_{D} / 2\right)$, with $l$ exponentially distributed with mean $L$.
}

during which a potential overhearer listens to a transmission. The energy $\widehat{P}_{R}\left(\bar{X}+T_{D}+T_{T}\right)+\widehat{P}_{T} T_{C}$ consumed to receive a packet includes the energy needed to listen to the wake-up preamble during $\bar{X}$ seconds, the energy to received the data packet, to turn-around into TX mode and to send an acknowledgement.

In expression (6), the first term represents the power consumed in DOZE state. The second term, $2 \theta \widehat{P}_{R}$, accounts for the time spent listening to the channel to cover the drift between the access point clock and the sensor node clock. The third term represents the power consumed to power-on and listen to the beacon of length $T_{C}$ every $T_{W}$ seconds. Finally, the fourth term accounts for the polling and reception of data packet every $L$ seconds, including the turn-around phases before to send the poll packet and to receive the data packet (see Fig. 4).

The transmission delay is defined as the time elapsed between the arrival of a packet at the access point and the end of its transmission to the destination sensor node. Under the low traffic assumption, it can be shown that the transmission delay with the WiseMAC and ZigBee protocol is respectively given by

$$
\begin{aligned}
D_{W i s e M A C}= & T_{D}+\frac{T_{W}}{2}\left(1-e^{-\frac{T_{W}}{4 \theta L}}\right) \\
& +2 \theta L\left(2-e^{-\frac{T_{D}}{4 \theta L}}-e^{-\frac{T_{W}}{4 \theta L}}\right)
\end{aligned}
$$

and

$$
D_{\text {ZigBee }}=\frac{T_{W}}{2}+2 T_{C}+2 T_{T}+T_{D}
$$

To derive expression (7), one has first to compute the transmission delay in different scenarios, depending of the value of the inter-arrival. Expression (7) is then obtained by averaging this composite function over the distribution of the inter-arrival.

Expression (8) is composed of the average time between the arrival of a packet and the start of the beacon transmission, the time to transmit the beacon and the time for the sensor node to send a poll and receive the data packet.

Expressions (6) and (8) have been derived without to take into account the potential collisions between poll packets, when several nodes compete to download data following the transmission of the beacon. For this reason, they will be evaluated for $T_{W} \leq 1 / \lambda$.

\subsection{Comparison}

The protocol performance is influenced by a number of system parameters. For the radio transceiver parameters, we will consider those of the WiseNET low power radio transceiver, as listed in Table 1. We consider the same radio transceiver for both the WiseMAC and the ZigBee MAC 
Table 1. System Parameters.

\begin{tabular}{|c|c||c|c|}
\hline Parameter & Value & Parameter & Value \\
\hline \hline$P_{Z}$ & $5 \mu \mathrm{W}$ & $T_{D}(50$ bytes $)$ & $16 \mathrm{~ms}$ \\
$P_{R}$ & $1.8 \mathrm{~mW}$ & $T_{C}(10$ bytes $)$ & $3.2 \mathrm{~ms}$ \\
$P_{T}$ & $27 \mathrm{~mW}$ & $N$ & 10 \\
$T_{S}$ & $0.8 \mathrm{~ms}$ & $L$ & $1000 \mathrm{~s}$ \\
$T_{T}$ & $0.4 \mathrm{~ms}$ & $\theta$ & $30 \mathrm{ppm}$ \\
$B$ & $25 \mathrm{kbps}$ & & \\
\hline
\end{tabular}
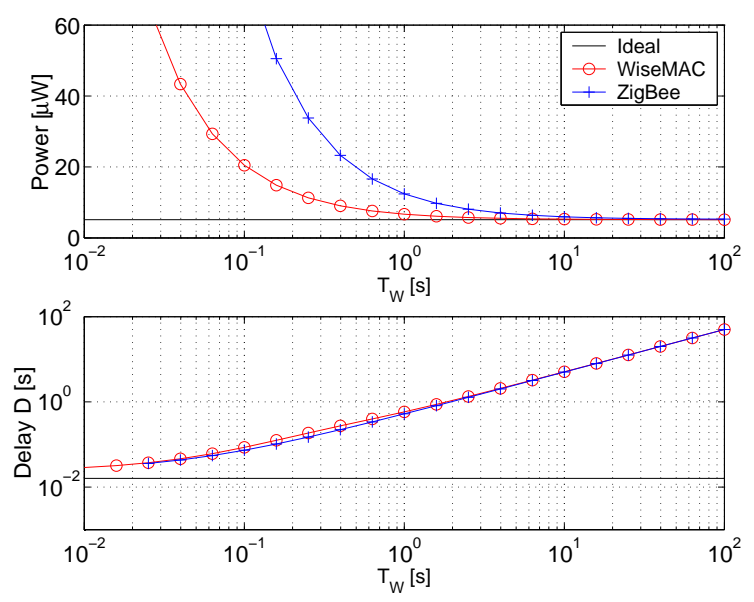

Figure 5. Power consumption and delay of WiseMAC and ZigBee as a function of the wake-up period $T_{W}(L=1000 \mathrm{~s})$.

protocols. WiseNET is the name of a project running at CSEM within which a low power radio transceiver targeted for sensor networks has been designed [17]. The remaining parameters have been chosen as follows: The frequency tolerance of the quartz is chosen to be $\theta= \pm 30 \mathrm{ppm}$, which corresponds to an inaccuracy of 2.6 seconds over one day. The length of the data and of the control packets are arbitrarily chosen to be respectively 50 and 10 bytes.

We begin by comparing the power consumption and delay performances of WiseMAC and $\mathrm{ZigBee}$ as a function of the protocol parameter $T_{W}$ for a fixed traffic $L$ and a fixed number of sensor nodes $N$. The choice of $T_{W}$ permits to trade a higher delay against a lower average power consumption. We consider $N=10$ sensor nodes and an inter-arrival per sensor node of $L=1000 \mathrm{~s}=16$ minutes. Recall that this traffic is supposed to consist of configuration and query commands sent by the sensor network controller. Large inter-arrivals make hence sense in this context.

Fig. 5 shows the power consumption $P$ and the latency $D$ as a function of the protocol parameter $T_{W}$. The horizontal line in the upper plot represents the power consumption of an ideal protocol, which is defined as the power needed

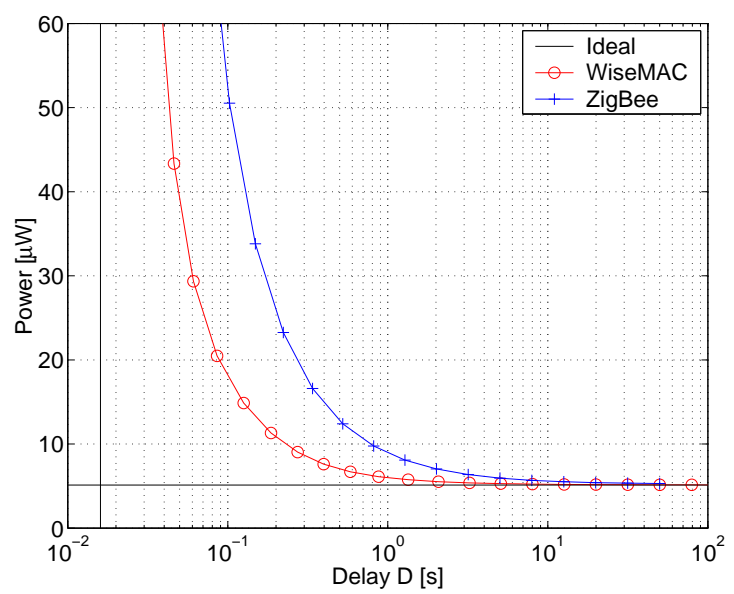

Figure 6. Power-delay characteristics of WiseMAC and ZigBee ( $L=1000 \mathrm{~s})$.

to receive the data, turn-around the transceiver and send the acknowledgement. In this case, we have $P_{I D E A L}=$ $P_{Z}+\frac{\widehat{P}_{R}\left(T_{S}+T_{D}+T_{T}\right)+\widehat{P}_{T} T_{C}}{L}=5.12 \mu \mathrm{W}$. It can be noticed that the incremental cost due to the data reception is only of $0.12 \mu \mathrm{W}$, a small value compared to $P_{Z}=5 \mu \mathrm{W}$.

In the lower plot, the horizontal line represents the minimum delay that would be obtained with an ideal protocol, i.e. $D_{I D E A L}=T_{D}=16 \mathrm{~ms}$. WiseMAC and ZigBee approach this limit for small values of $T_{W}$, but at a high power cost. For large values of $T_{W}$, the two curves converge to the average waiting delay $T_{W} / 2$.

Using both plots, a designer can choose a value for the parameter $T_{W}$, making a trade-off between the consumed power and the average transmission delay. To compare the protocols between them, one can combine both plots and draw the power-delay characteristics for a varying $T_{W}$, as shown in Fig. 6. The horizontal line represents the ideal power consumption and the vertical line the ideal delay.

One can see that WiseMAC consumes clearly less power than ZigBee. The cost of receiving data being relatively small when $L=1000 \mathrm{~s}$, this can be understood by comparing the basic cost of their respective wake-up scheme. With an infinitely low traffic $(L \rightarrow \infty)$, the power consumption of WiseMAC and ZigBee becomes respectively

$$
\begin{gathered}
\lim _{L \rightarrow \infty} P_{W i s e M A C}=P_{Z}+\frac{\widehat{P}_{R}\left(T_{S}+1 / B\right)}{T_{W}} \\
\lim _{L \rightarrow \infty} P_{Z i g B e e}=P_{Z}+2 \theta \widehat{P}_{R}+\frac{\widehat{P}_{R}\left(T_{S}+T_{C}\right)}{T_{W}}
\end{gathered}
$$

With WiseMAC, the transceiver powers up every $T_{W}$ to listen to the channel during the duration of a radio symbol. With ZigBee, the transceiver periodically receives a beacon. As the duration of a beacon is always larger than the 


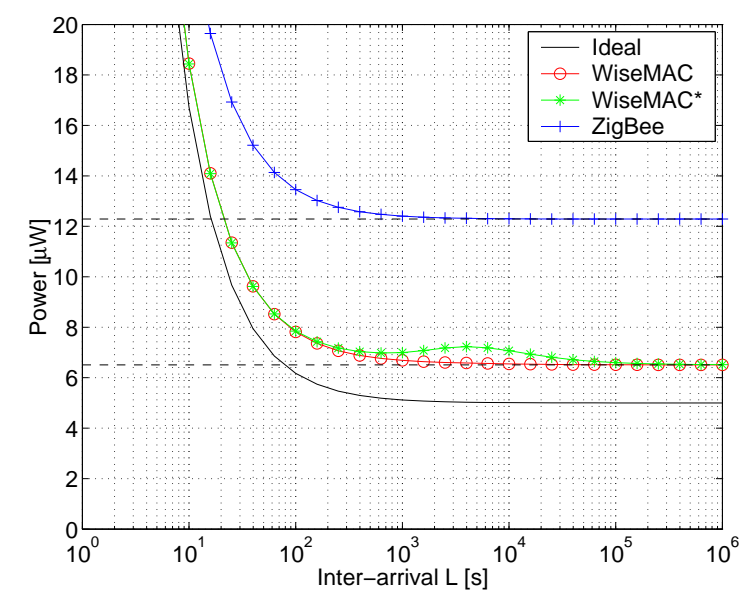

Figure 7. Power consumption of WiseMAC and ZigBee as a function of the inter-arrival $L\left(T_{W}=1 \mathrm{~s}\right)$.

duration of a modulation symbol, the wake-up scheme of WiseMAC consumes less than the one of ZigBee.

Assuming that, based on the requirements of some application, a designer would choose to have an average delay of 0.5 second (and a maximum delay of 1 second). This would imply to select $T_{W}=1 \mathrm{~s}$. Receiving one message every $L=1000 \mathrm{~s}$, the power consumption of WiseMAC would amount to $7 \mu \mathrm{W}$, only $2 \mu \mathrm{W}$ above the DOZE power consumption. When powered by a single alkaline battery of 2.6 Ah capacity with a constant power leakage of $27 \mu \mathrm{W}$, this power consumption would translate into a lifetime of 8 years (without uplink traffic). See [13] for a description of the battery model. For the same delay, the power consumption of the ZigBee protocol amounts to $13 \mu \mathrm{W}$, i.e. $85 \%$ more than WiseMAC. To consume $7 \mu \mathrm{W}$ with the ZigBee protocol, the average delay must be extended to 2.5 seconds, i.e. five times more.

\subsection{Sensitivity Analysis}

We have considered in the previous section a constant traffic $L=1000$ and a constant number of sensor nodes $N=10$. In this section, the impact of the variation of those parameters on the performance of WiseMAC and ZigBee is analyzed, considering a fixed wake-up period $T_{W}=1$. We will see the importance of the repetition of the data frames in long wake-up preamble, through a comparison with the performance of WiseMAC*, a sub-optimal version of the protocol where long wake-up preambles are not composed of repetition of data frames, but simply of alternating bits. This version was considered in [14].

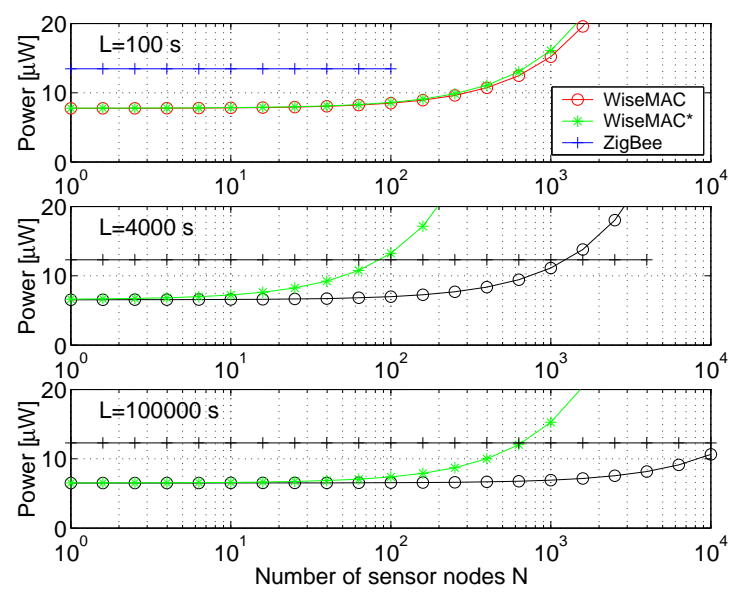

Figure 8 . Scalability of WiseMAC with and
without repetition of data frames in long
wake-up preambles $\left(T_{W}=1 \mathrm{~s}\right)$.

Varying traffic. Fig. 7 shows the power consumption of WiseMAC and ZigBee as a function of the inter-arrival $L$. It can be observed that WiseMAC remains superior to ZigBee in both high and low traffic conditions. When $L$ is large (low traffic), the power consumption of WiseMAC approaches the one of the ideal protocol. This property is brought by the proportionality between the wake-up preamble length and the inter-arrival. The per-packet overhead decreases as the traffic increases. When $L$ increases (decreasing traffic), the power consumption of WiseMAC decreases monotonically towards (9).

Fig. 7 shows also the power consumption of WiseMAC*, It can be seen that the power consumption of WiseMAC* is larger than the one of WiseMAC for medium traffic. This is caused by the cost of overhearing. Overhearing is maximized, with Poisson traffic, for $L \approx \frac{T_{W}}{8 \theta} \approx 4000$. For lower values of $L$, the length of the wake-up preamble is smaller than $T_{W}$ and overhearing is mitigated in a probabilistic way. For higher values of $L$, the power consumption of WiseMAC* converges again to the power consumption of WiseMAC as the power consumption of data packet reception (including overhearing) becomes negligible. With $N=10$ nodes, the difference between the power consumption of WiseMAC and WiseMAC* is small. But as the overhearing term is proportional to $N$, this becomes important when considering scalability.

Scalability. Fig. 8 shows the power consumption of WiseMAC, of WiseMAC* ${ }^{*}$ and of ZigBee, as a function of the number of nodes $N . L$ is kept constant. An increasing $N$ results in an increasing global traffic. It can be seen in expression (6) than the power consumption of ZigBee is independent of $N$. ZigBee does not cause any overhearing 
and therefore scales perfectly ${ }^{5}$. On the contrary, WiseMAC suffer from an overhearing component (last term in expression (3)). As the overhearing component is proportional to the number of nodes, this will limit its scalability. As can be seen in Fig. 8, WiseMAC remains however more energy efficient than ZigBee up to hundreds of nodes, a number above the needs of most applications. Fig. 8 also shows the importance of repeating data frames in long preambles. It can be seen that WiseMAC* scales poorly as compared to WiseMAC, in particular for a medium traffic of $L=4000 \mathrm{~s}$.

\section{Conclusion}

This paper has proposed the use of WiseMAC, a protocol based on synchronized preamble sampling, for the downlink of infrastructure sensor networks. The power consumption-delay trade-off was analyzed in low traffic conditions. A comparison was made with the power save protocol used in the IEEE 802.15.4 ZigBee standard. It was shown that WiseMAC can provide a significantly lower power consumption for the same delay. Using the WiseMAC protocol with the WiseNET transceiver, a sensor node consumes $7 \mu \mathrm{W}$ to receive 50 bytes data packets every 1000 seconds with an average latency of 0.5 seconds. When using the power save protocol of ZigBee, reaching the same latency would cause a power consumption of $13 \mu \mathrm{W}$, i.e. $85 \%$ more than with WiseMAC.

\section{References}

[1] J. Rabaey et al., "Picoradio supports ad hoc ultra-low power wireless networking," IEEE Computer Magazine, pp. 42-48, July 2000.

[2] Chipcon, "CC1010 Integrated RF Transceiver and Microcontroller," Brochure, Sept 2002.

[3] I. F. Akyildiz, W. Su, Y. Sankarasubramaniam, and E. Cayirci, "Wireless sensor networks: A survey," IEEE Communications Magazine, vol. 40, no. 8, pp. 102-114, August 2002.

[4] A. Mainwaring, J. Polastre, R. Szewczyk, D. Culler, and J. Anderson, "Wireless Sensor Networks for Habitat Monitoring," in Proc. Int. Workshop on Wireless Sensor Networks and Applications (WSNA), Sept 2002.

[5] W. Ye, J. Heidemann, and D. Estrin, "An Energy-Efficient MAC Protocol for Wireless Sensor Networks ," in Proc. IEEE INFOCOM Conf., 2002.

[6] L. Kleinrock and F. A. Tobagi, "Packet Switching in Radio Channels: Part 1-Carrier Sense Multiple-Access Modes and Their Throughput-Delay Characteristics," IEEE Transactions on Communications, vol. 23, no. 12, pp. 1400-1416, 1975.

\footnotetext{
${ }^{5}$ Above the validity limit of expression (6), i.e. for $N>L / T_{W}$, potential collisions between poll packets may limit the scalability of ZigBee.
}

[7] V. Bharghavan et al., "MACAW: A Media Access Protocol for Wireless LAN's," in Proc. ACM SIGCOMM Conf., 1994.

[8] J.-C. Chen, K. M. Sivalingam, and P. Agrawal, "Performance Comparison of Battery Power Consumption in Wireless Multiple Access Protocols," Wireless Networks, vol. 5, pp. 445-460, 1999.

[9] C. Schurgers, V. Tsiatsis, and M. B. Srivastava, "STEM: Topology Management for Energy Efficient Sensor Networks," in Proc. IEEE Aerospace Conf., vol. 3, Mar 2002, pp. 1099-1108.

[10] ITU-R, "M.584-2 Codes and formats for radio paging," Nov 1997.

[11] IEEE, "Wireless Medium Access Control (MAC) and Physical Layer (PHY) specifications for Low Rate Wireless Personal Area Networks (LR-WPANS)," IEEE 802.15.4-2003, 2003.

[12] A. El-Hoiydi, J.-D. Decotignie, C. Enz, and E. Le Roux, "Poster Abstract: WiseMAC, An Ultra Low Power MAC Protocol for the WiseNET Wireless Sensor Network," in Proc. 1st ACM SenSys Conf., November 2003, pp. 302-303.

[13] A. El-Hoiydi, "Spatial TDMA and CSMA with Preamble Sampling for Low Power Ad Hoc Wireless Sensor Networks," in Proc. IEEE Int. Conf. on Computers and Communications (ISCC), Taormina, Italy, July 2002, pp. 685-692.

[14] A. El-Hoiydi, J.-D. Decotignie, and J. Hernandez, "Low Power MAC Protocols for Infrastructure Wireless Sensor Networks," in Proc. European Wireless (EW'04), Barcelona, Spain, February 2004, pp. 563-569.

[15] IEEE, "Wireless LAN Medium Access Control (MAC) and Physical Layer (PHY) Specifications," IEEE 802.11, ISO/IEC 8802-11:1999, 1999.

[16] A. El-Hoiydi and J.-D. Decotignie, "Low Power Downlink MAC Protocols for Infrastructure Wireless Sensor Networks," submitted for publication, 2004.

[17] T. Melly, E. Le Roux, F. Pengg, D. Ruffieux, N. Raemy, F. Giroud, A. Ribordy, and V. Peiris, "WiseNET: Design of a Low-Power RF CMOS Receiver Chip for Wireless Applications," CSEM Scientific and Technical Report, p. 25, 2002. 